\section{First trimester bleeding}

Shweta Bhatt, MD

MAYO
CLINIC
Fj

\section{Causes}

- Abortion

- Ectopic pregnancy

- Molar pregnancy (GTD)

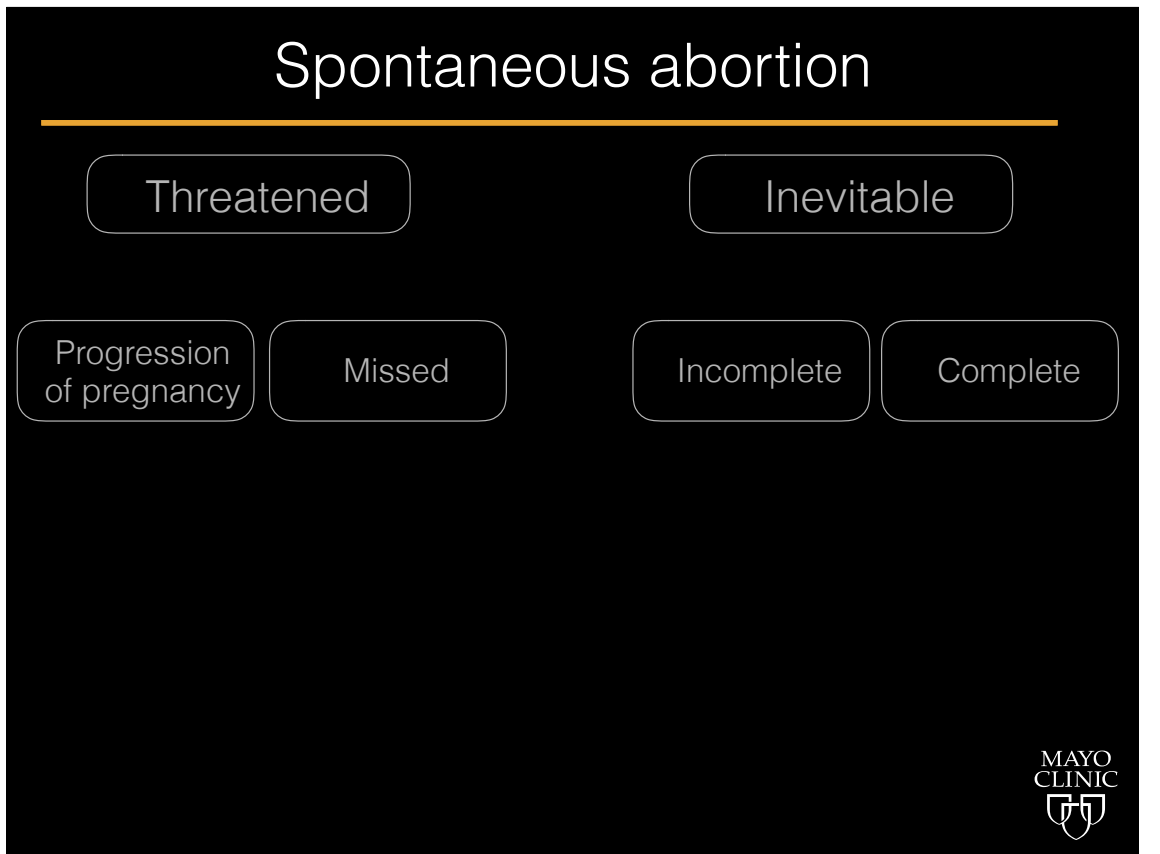

\title{
Spontaneous abortion
}

Causes:

-Chromosomal abnormalities

-Defective implantation

-H Mole

-Fibroids

- Maternal infections

- Medical disorders - DM, HTN, etc

- Uterine abnormalities - septate/bicornuate/

retroverted etc

-Environmental factors - smoking, alcohol

- Maternal age >30yrs

- Paternal factors

-Immunological 


\section{Spontaneous abortion}

Threatened abortion

Signs and symptoms:

- Abdominal pain

-Vaginal bleeding - scant (spotting)

- Cervical os closed

- Uterus non tender

Ultrasound performed frequently to confirm viability

\section{Spontaneous abortion}

\section{Missed abortion}

Signs and symptoms:

- No abdominal pain

- No/minimal vaginal bleeding

- Cervical os closed

-Uterus smaller

Ultrasound - Non viable fetus

\section{Spontaneous abortion}

\section{Spontaneous abortion}

Incomplete abortion

Signs and symptoms

- Severe abdominal pain

- Heavy vaginal bleeding

- Cervical os open

- Uterus tender

-Retained products of conception

- Static or slow decline in hCG

- Ultrasound shows RPOC
Signs and symptoms:

- Severe abdominal pain

- Heavy vaginal bleeding

- Cervical os open

- Uterus smaller and /or tender
Inevitable abortion 
Inevitable abortion: Abortion in progress
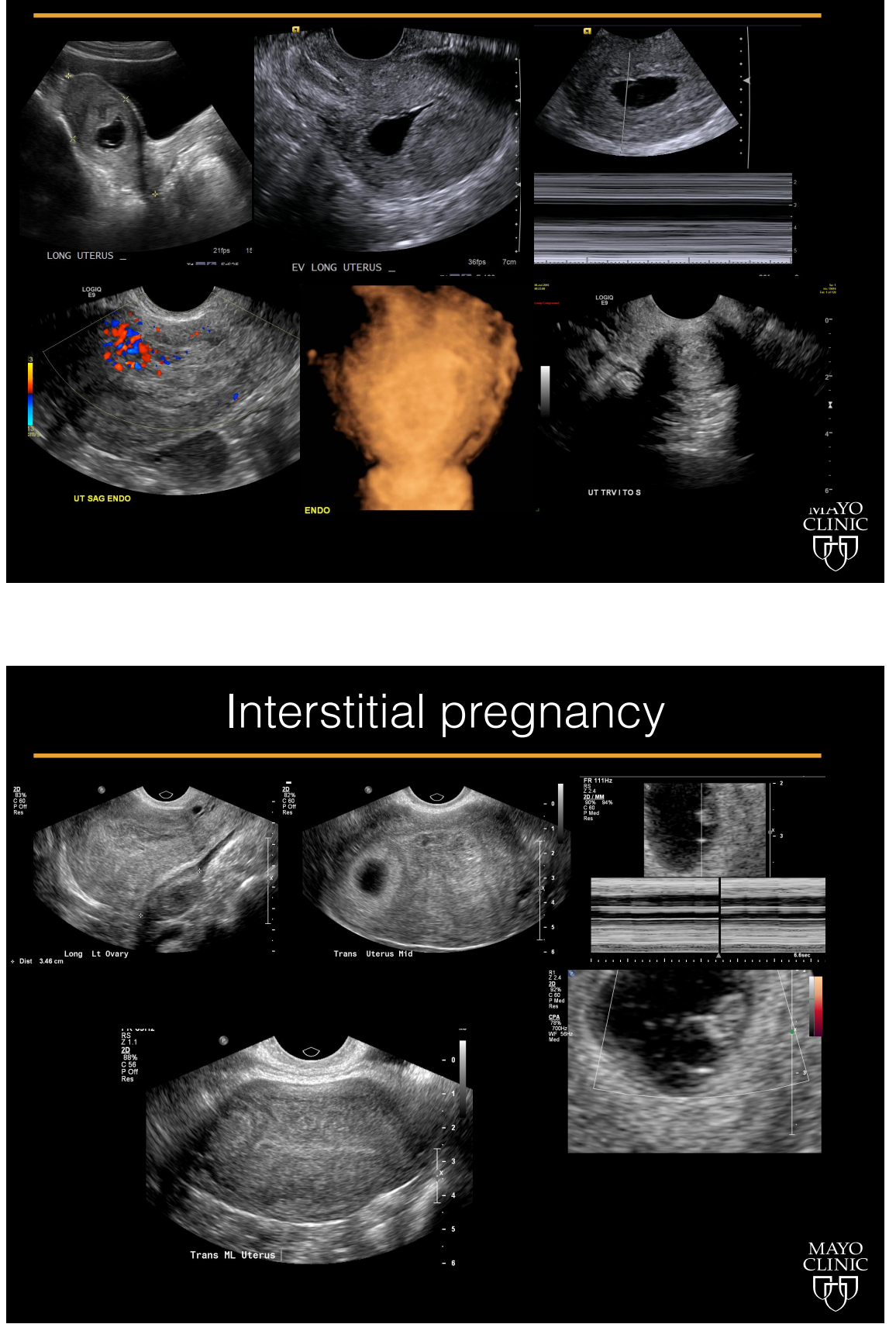

Inevitable Ab

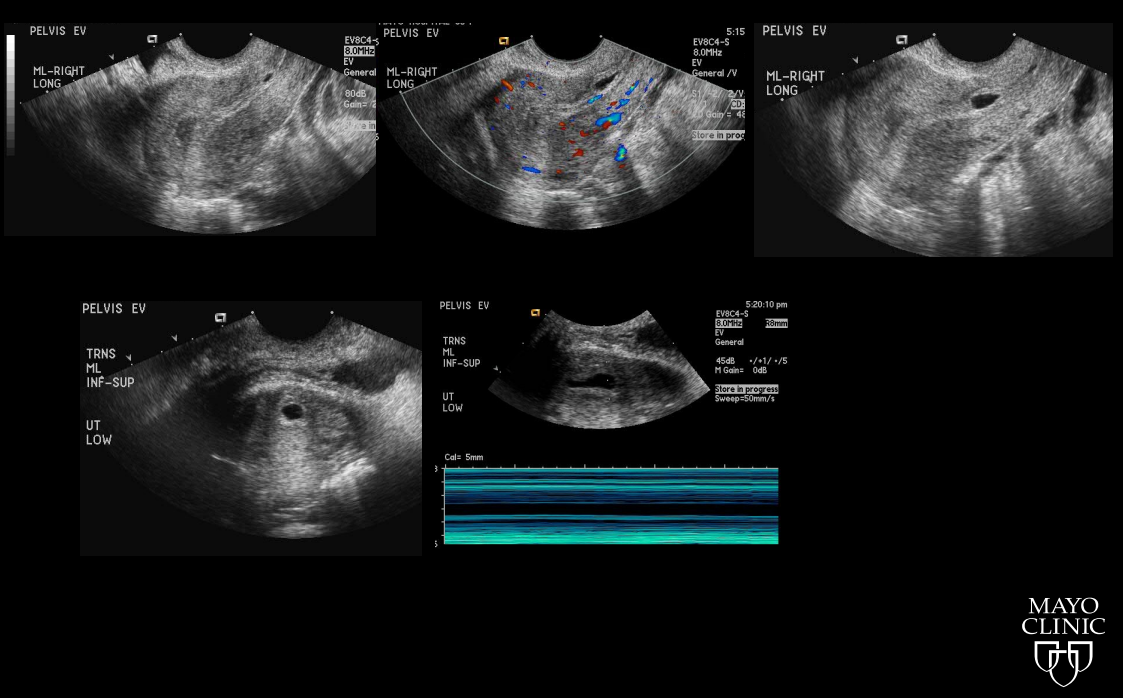

\section{Blighted ovum}

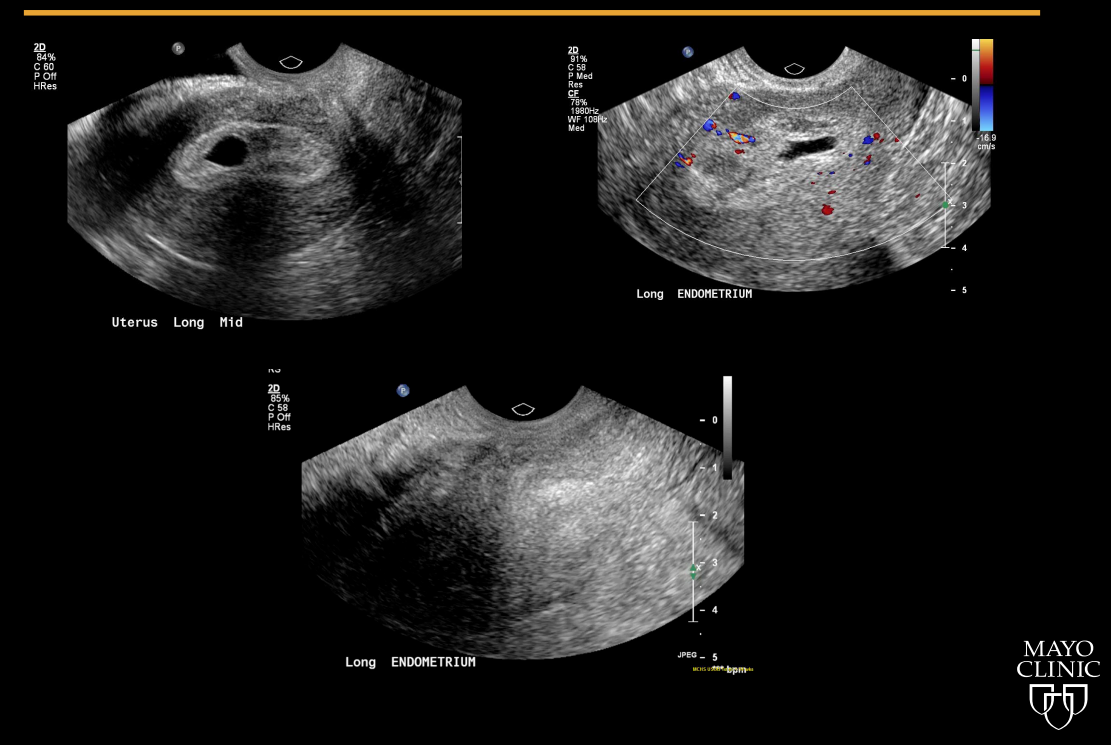


Impending abortion (in progress)
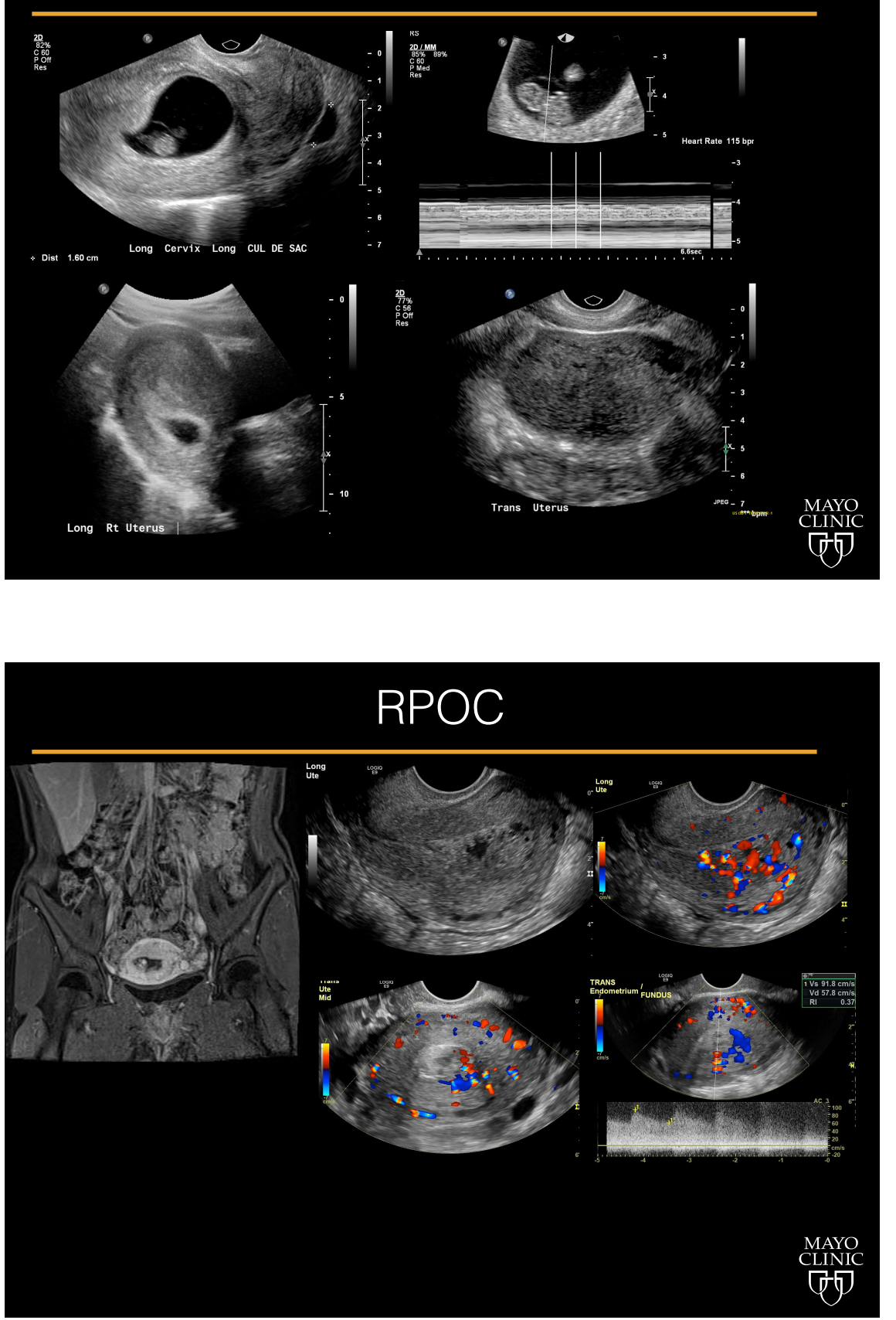

RPOC

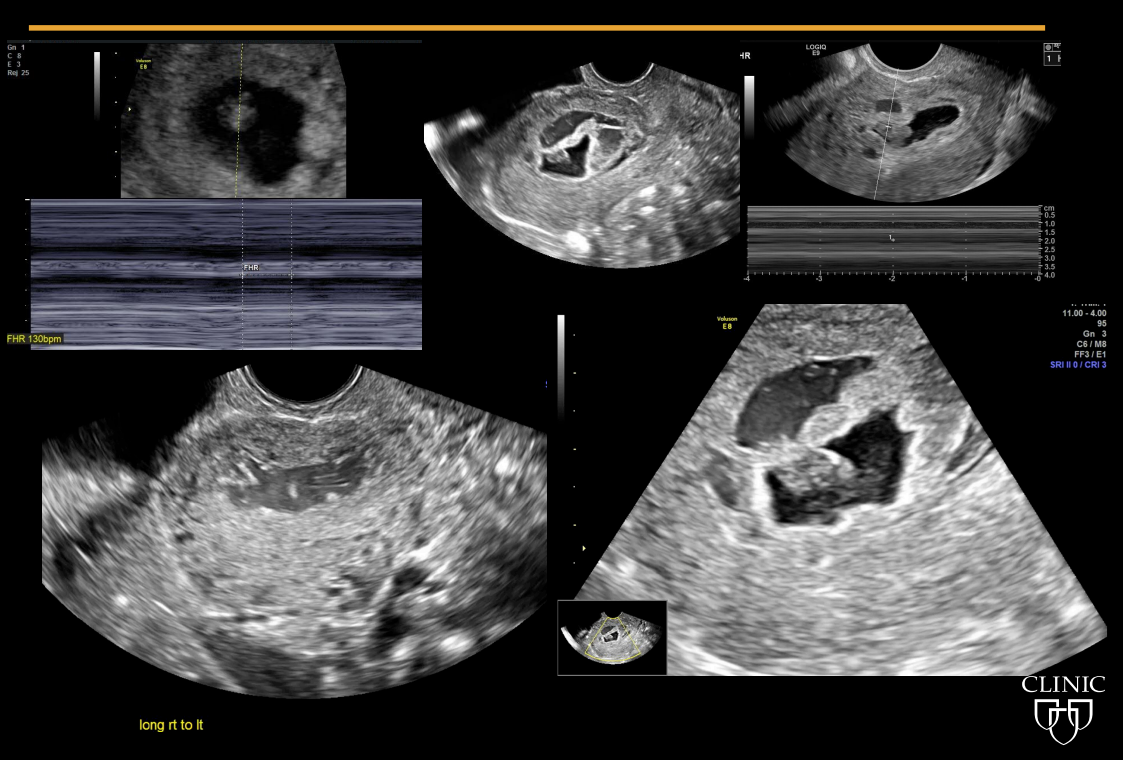

Missed abortion

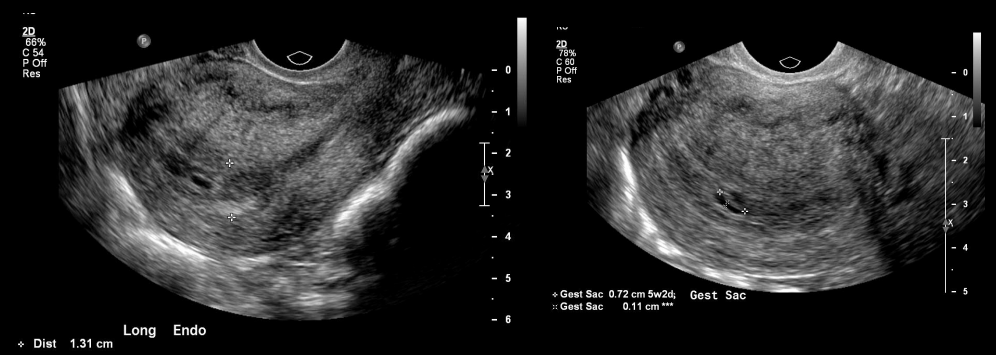




\section{Spontaneous abortion}

\section{Complete abortion}

Signs and symptoms:

-Diminishing or absent abdominal pain

-No vaginal bleeding

- Cervical os closed

-Uterus firm

Normal uterus on Ultrasound
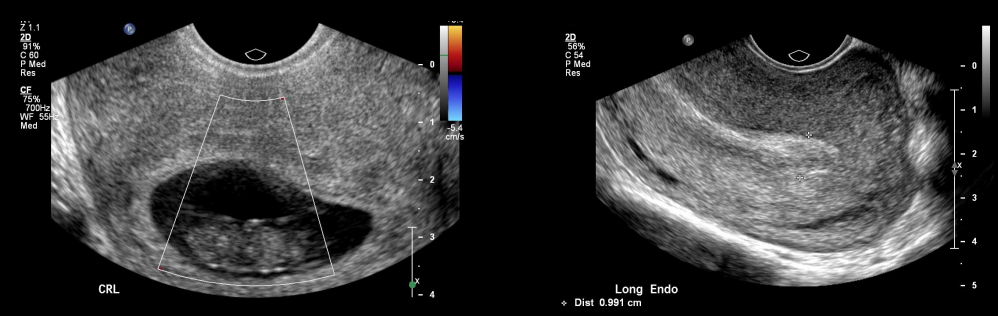

- Ditut osog cong Endo

\section{Gestational trophoblastic disease}

Hydatiform mole

\section{Choriocarcinoma}

Signs and symptoms:

- Exaggerated signs of pregnancy - high hCG -Passage of vesicles

-Bleeding

- Larger than expected uterus

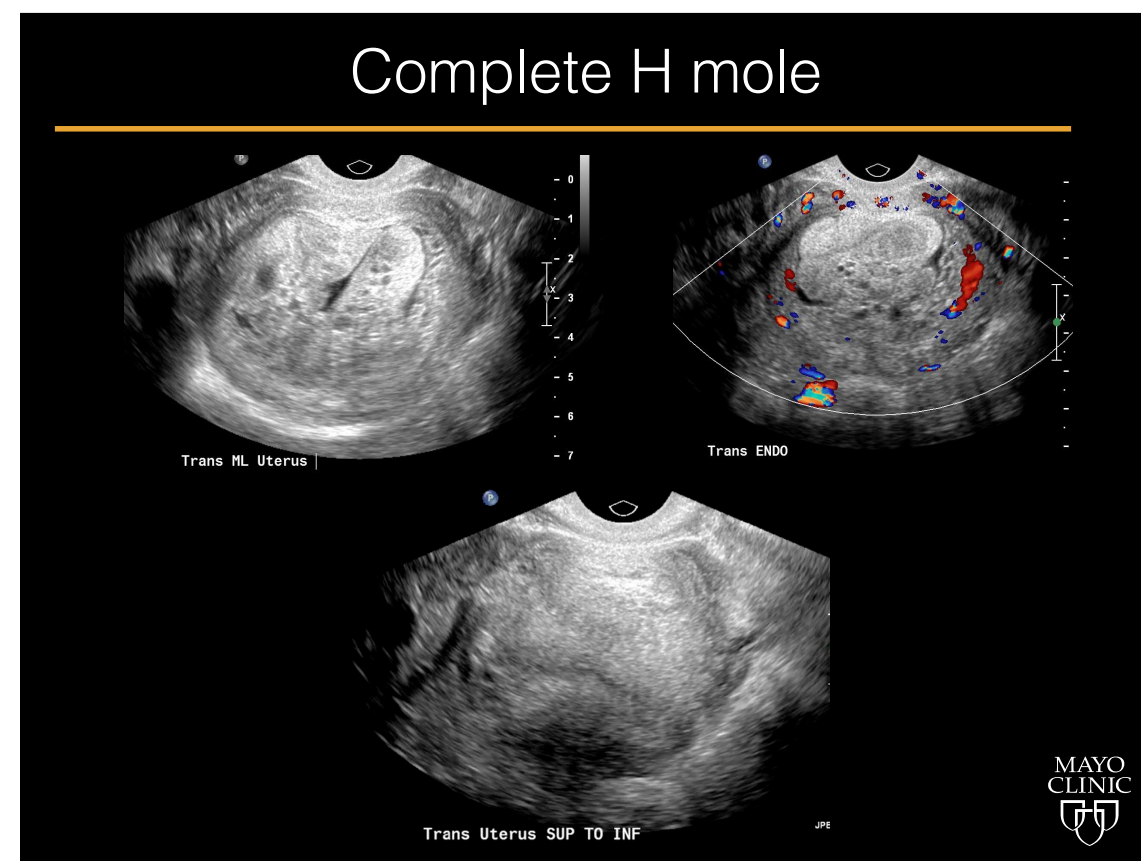



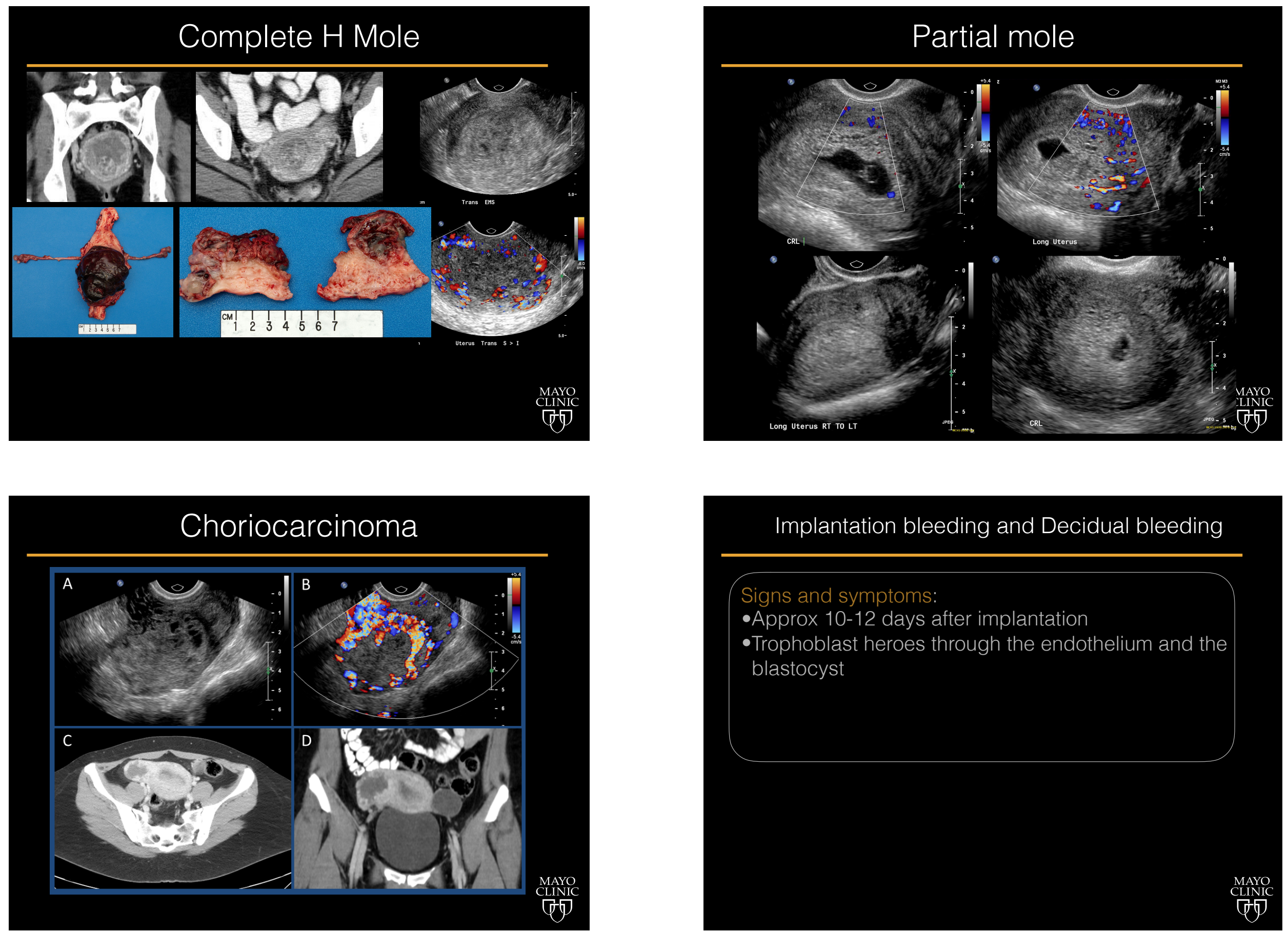

Implantation bleeding and Decidual bleeding

Signs and symptoms:

-Approx 10-12 days after implantation

- Trophoblast heroes through the endothelium and the blastocyst 
Ectopic pregnancy

- Defined as an extrauterine pregnancy

- Leading cause of pregnancy related mortality in the first trimester

$9 \%$ of pregnancy related deaths

- Incidence of $1.9 \%$

- John Bard described the first case with surgical outcome in 1759 in NY
Risk factors for Ectopic pregnancy

- Prior gynecologic infection

- Infertility

- Prior ectopic pregnancy

- History of intrauterine contraceptive device use

- Prior gynecologic surgery

- History of prior placenta previa

- In vitro fertilization (IVF)

- Congenital uterine or tubal anomalies

- Smoking

- Prior DES exposure
Clinical presentation

- Less than $50 \%$ present with classic triad of pelvic pain, vaginal bleeding and amenorrhea

- Other symptoms include those of early pregnancy
Human Chorionic Gondaotropin (HCG)

- Glycoprotein hormone produced initially by the embryo during first week and later by syncytiotrophoblasts within the placenta

- Sustains progesterone production by the corpus luteum

- HCG levels peak at 9-11 weeks (30-100IU/ $\mathrm{mL}$ )

- Declines to 5-10 IU/mL at week 20 
Common locations of ectopic pregnancy

- $95 \%$ of ectopic pregnancies are tubal, occurring mostly in the isthmus or ampulla

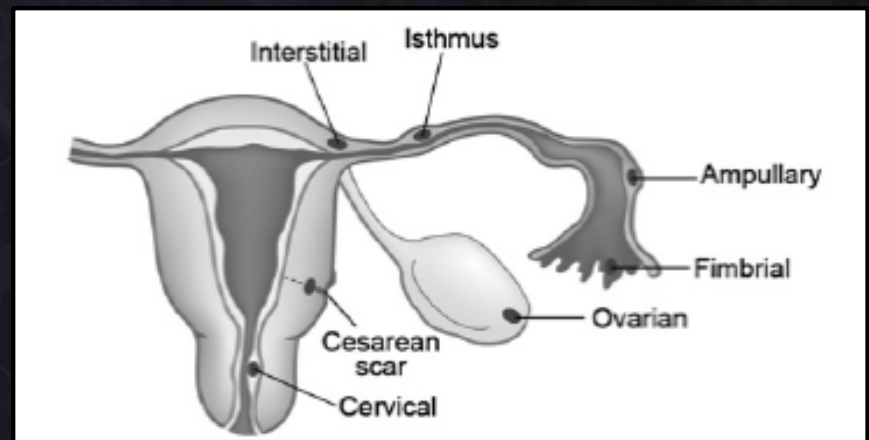

RIT 2017

Uterine findings of ectopic pregnancy

- Approximately $26 \%$ of patients have a normal pelvic ultrasound

- Thick decidual cast without a gestational sac

- Pseudogestational sac (10\%): intrauterine fluid surrounded by thick decidual reaction

- Trilaminar endometrium

- Thin walled decidual cyst at junction of endometrium and myometrium

\section{Locations}

95\% tubal, $5 \%$ other

Ampulla (75-80\%)

Isthmus (10-15\%)

Fimbria (5\%)

Interstitial Comusl region of uterine fundus ( $<5 \%)$

Ovary $(<1 \%)$

Cervix $(0.1 \%)$

Abdominal pregnancy - intraperitoneal surface or between the leaves of the broad ligament $(0.03 \%)$

RIT 2017

- Adnexal mass containing yolk sac or embryo

- Tubal ring sign: hyperechoic ring surrounding gestational sac

- Ring of fire sign: peripheral hypervascularity surrounding gestational sac

- Pelvic free fluid in cul de sac

- Pelvic hemorrhage: $90 \%$ (+) predictive value

- Simple adnexal cyst

- Solid adenxal mass 


\section{Common findings in ectopic pregnancy}

a. Tubal ring sign - 2nd most common finding

b. Pelvic hemorrhage

c. Pseudogestational sac

d. Extraovarian adnexal mass - Found in more than $89 \%$ patients with ectopic pregnancy on US

Tubal ring sign : variable appearance

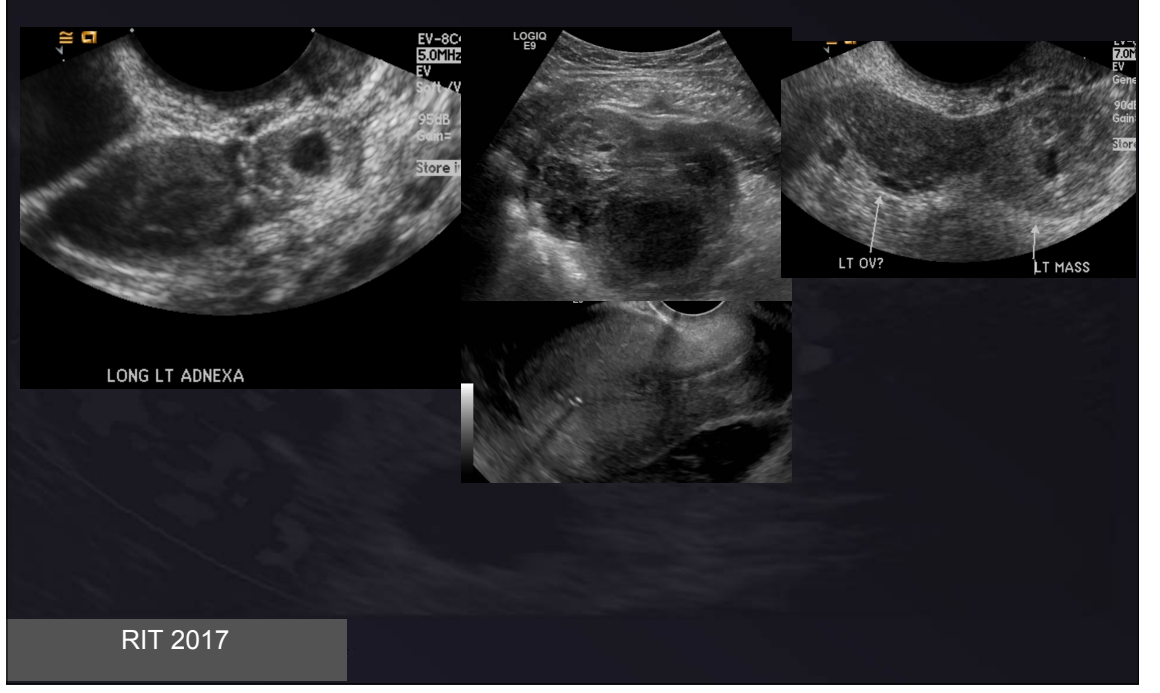

\section{Tubal Ring Sign}

Extraovarian mass with tubal ring sign (arrowheads) separate from left ovary (arrow)

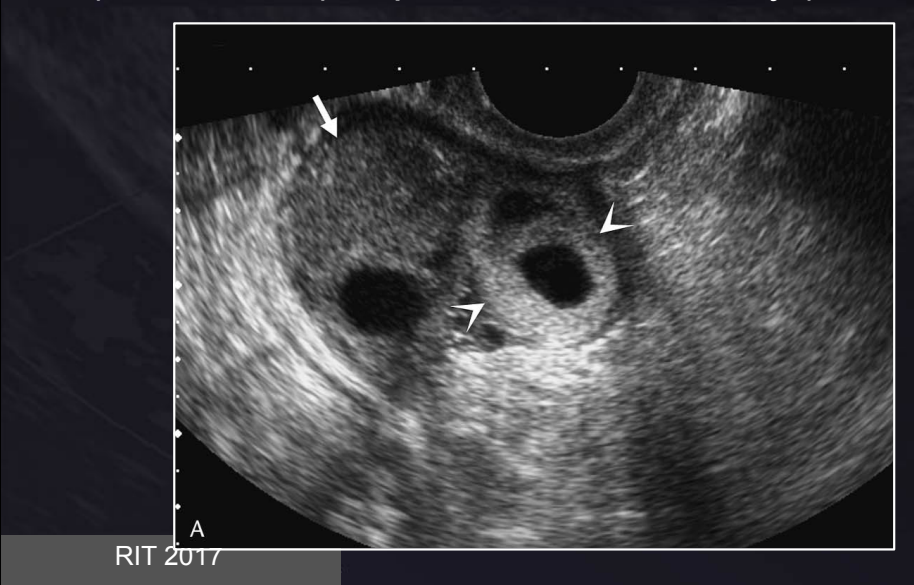

\section{Mimics}

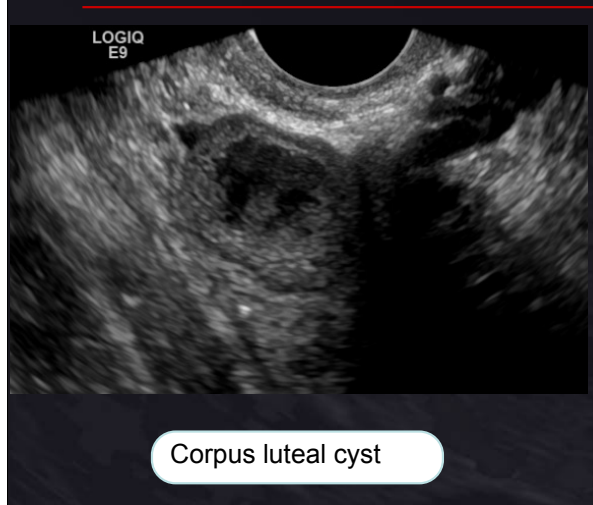




\section{Ring of Fire Sign}

Tubal pregnancy demonstrating Ring of Fire sign, with peripheral vascularity on color flow Doppler and low resistance high peak systolic velocity
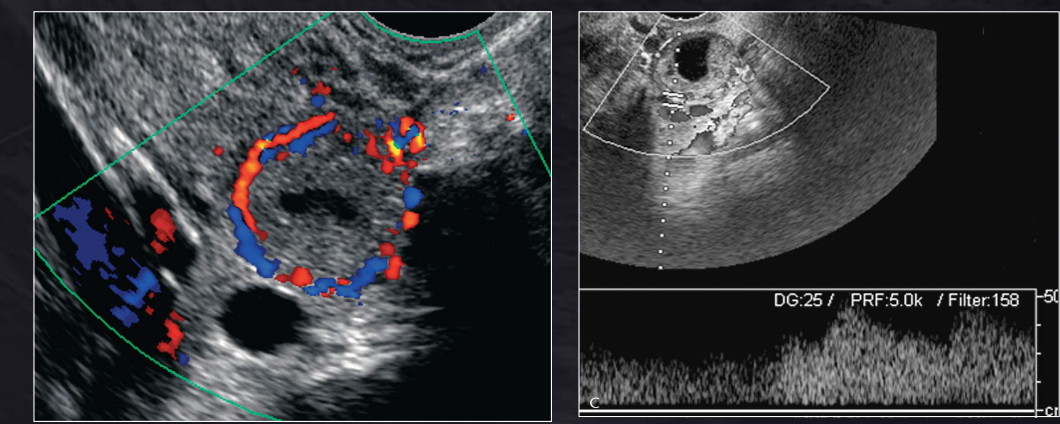

RIT 2017

Pseudogestational sac

Comparison of pseudogestational sac $(A)$ and intrauterine pregnancy (B) containing gestational sac, surrounded by thick hyperechoic layer (arrow heads decidua capsularis).

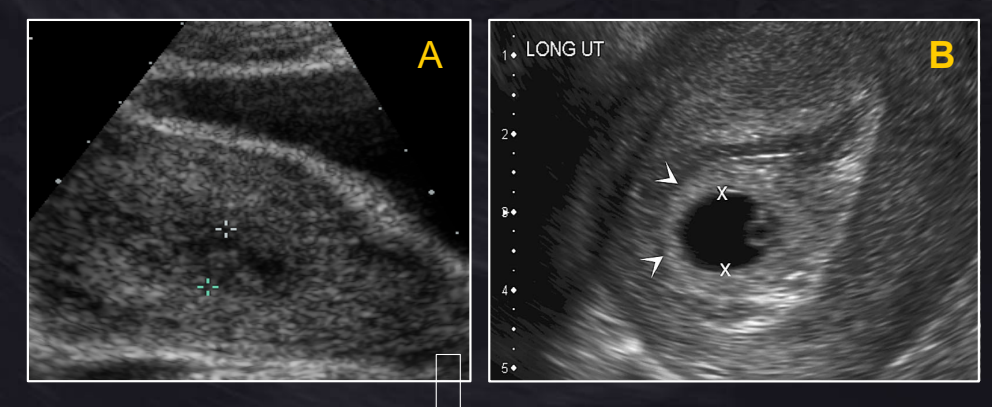

RIT 2017

\section{Ring of fire: non specific}

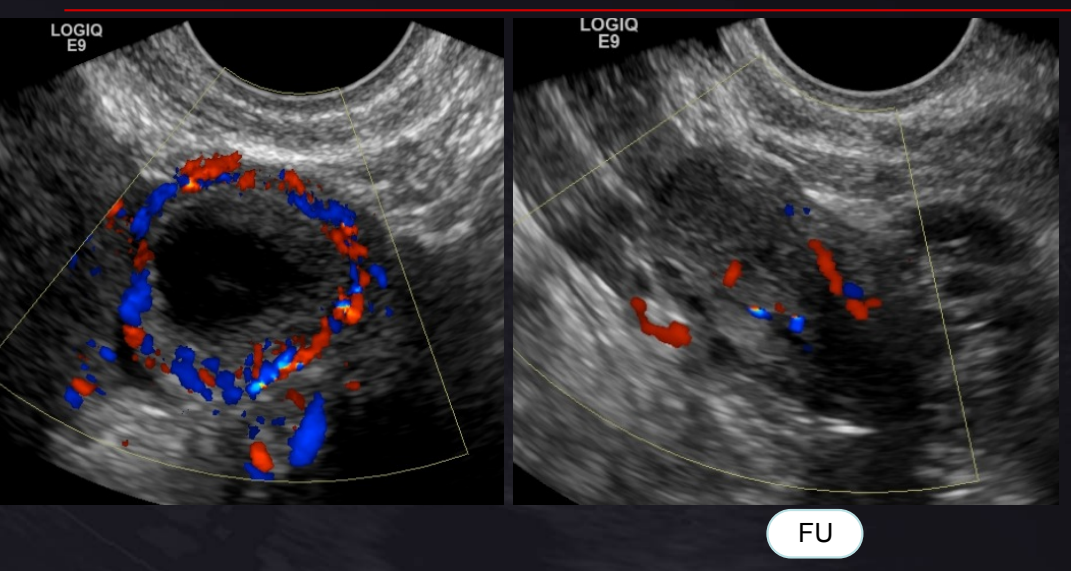

RIT 2017

\section{Pseudogestational sac}

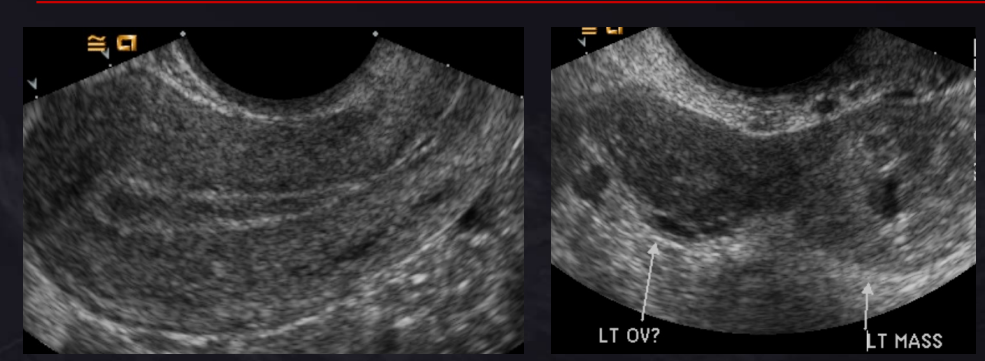




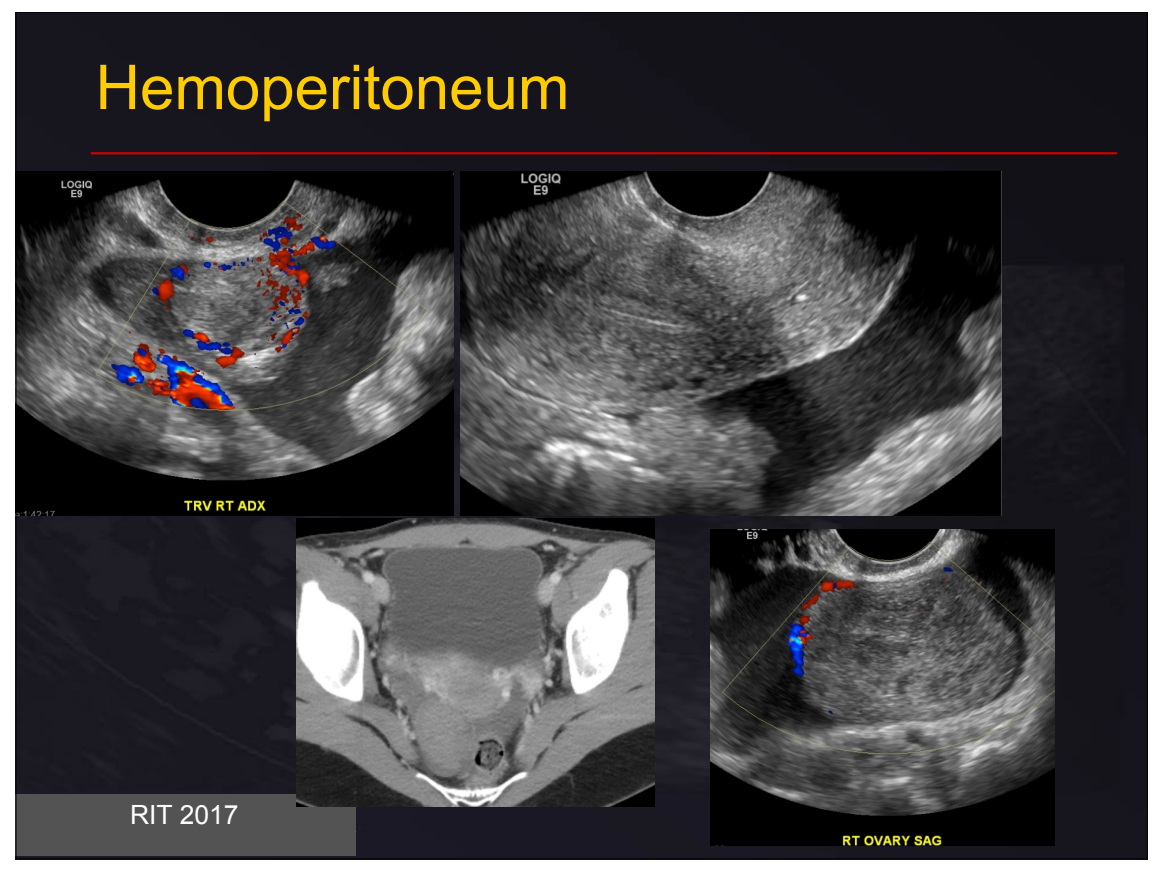

Mimic : hemorrhagic cyst rupture
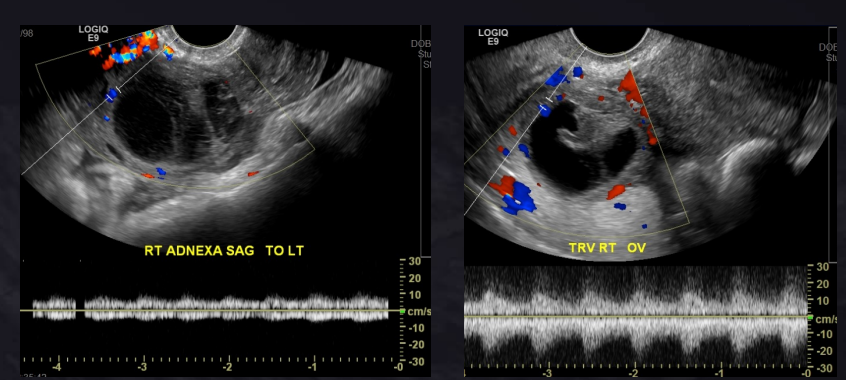

Mimic: Ovarian capsular artery bleed

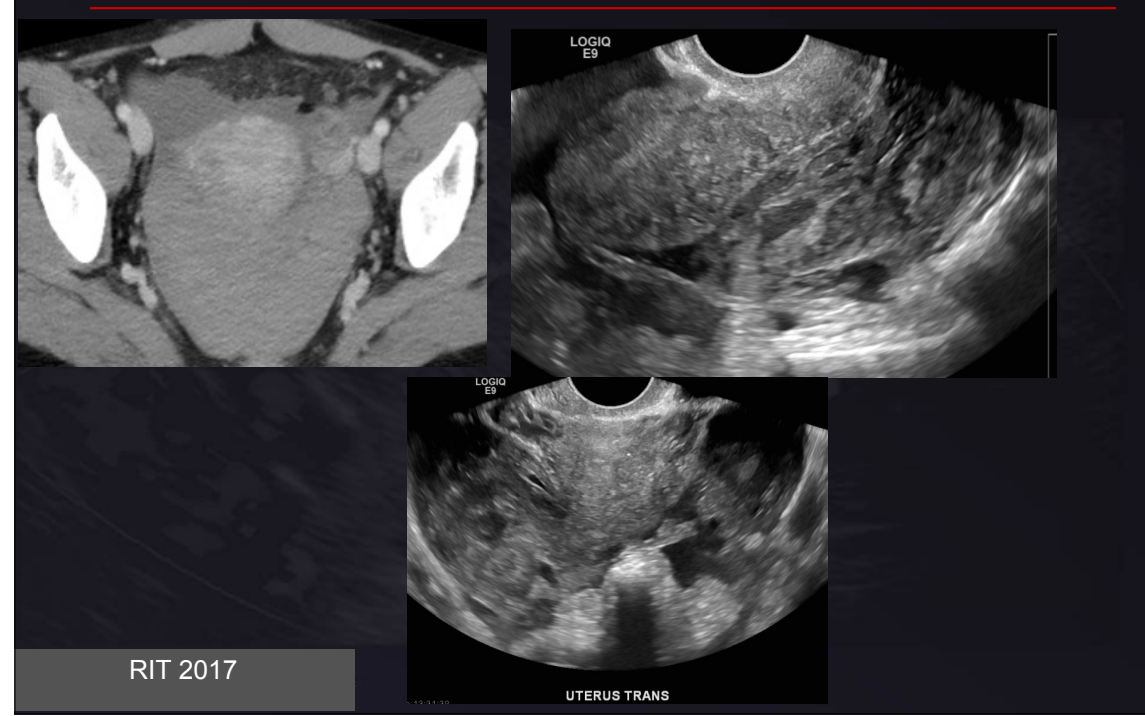

IUD and ectopic

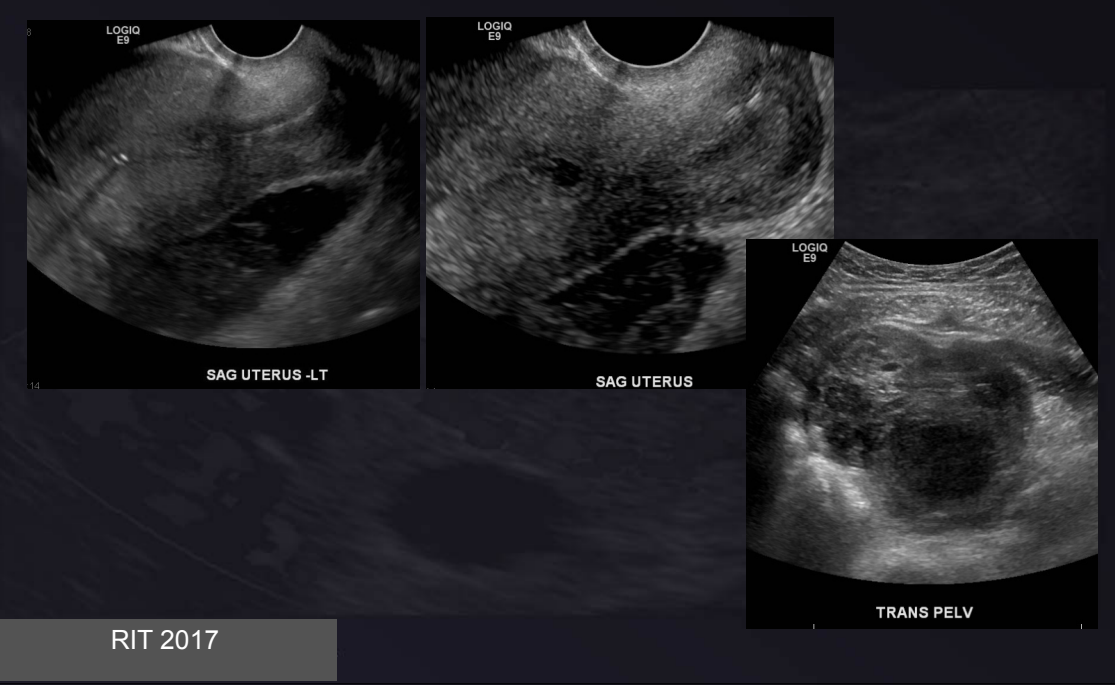




\section{IUD and ectopic}

IUD does not increase the risk of ectopic pregnancy.

- US criteria proposed by Timor-Tisch et al [1992] - Empty uterine cavity

- Eccentric gestational sac located $>1 \mathrm{~cm}$ from lateral uterine wall

- Myometrial mantle sign: Thin myometrial layer $(<5$ $\mathrm{mm}$ ) surrounding sac
Interstitial/cornual pregnancy

- Implantation of gestational sac in intramural portion of proximal fallopian tube

- Rare, incidence of $2-4 \%$

- Often early rupture and severe hemorrhage (>12 weeks)

- Associated with high mortality/morbidity

\section{Interstitial pregnancy (cont'd)}

- Eccentrically located gestational sac (arrow) near fundus (Fu) - Bulging sign

- Interstitial line: echogenic line (arrow) extending from endometrium to gestational sac

- $80 \%$ sensitive, $98 \%$ specific

- Hypo echoic myometrium separating the ectopic sac from the endometrial canal

- 3D shows the sac in the interstitial potion of Fallopian tube.

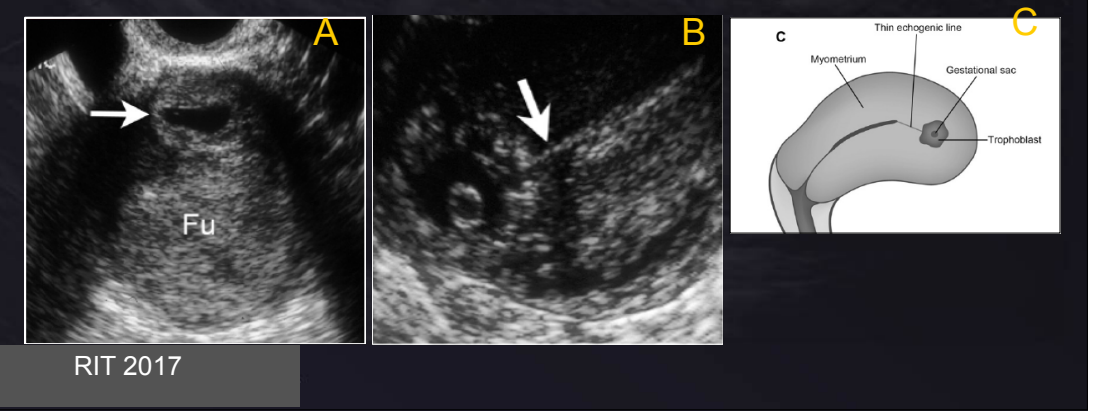




\section{Angular pregnancy}

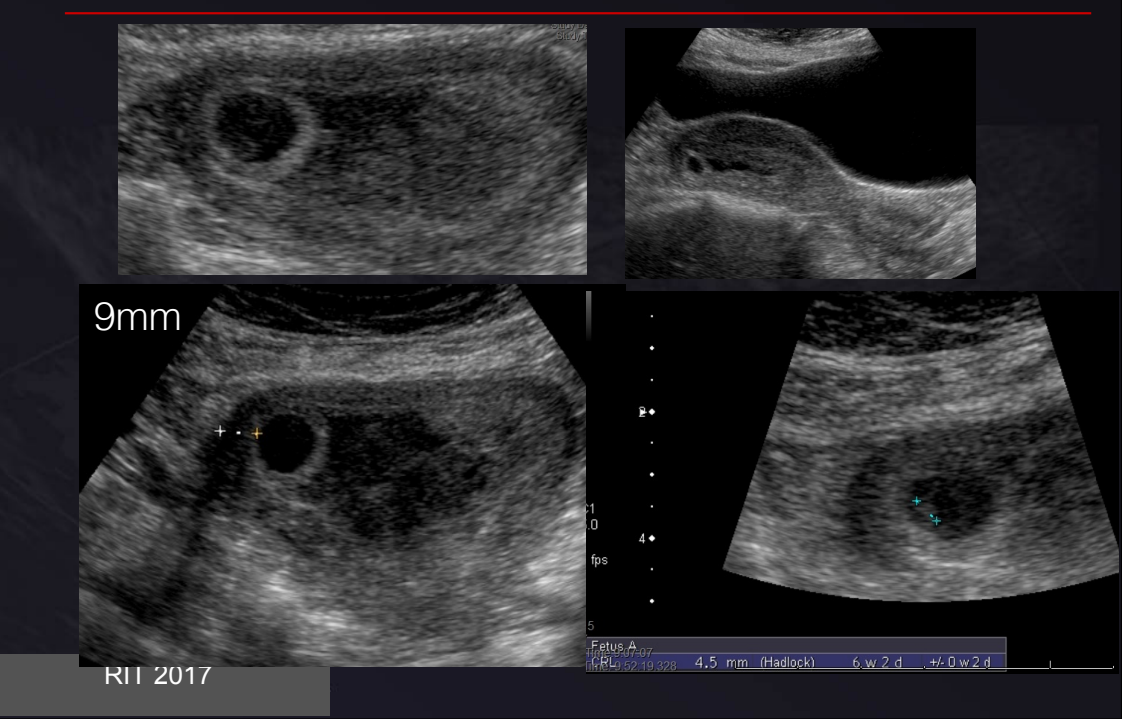

Cervical pregnancy

- US criteria proposed by Kung et al. [2004]

- gestational sac within cervix

- Empty uterine cavity

- Normal endometrial stripe

- Hourglass uterine with bulging cervical canal

- Active cardiac activity below internal os

\section{Angular versus Interstitial pregnancy}

\begin{tabular}{|c|c|}
\hline IUP/Angular Pregnancy & Interstitial \\
\hline $\begin{array}{l}\text { - IUP implanted eccentrically in } \\
\text { one of the lateral angles/ } \\
\text { corners of uterine cavity } \\
\text { - Large broad based } \\
\text { connection with endometrium } \\
\text { - Outer border surrounded by } \\
\text { myometrium ( }>5 \mathrm{~mm} \text { ) } \\
\text { - 3D ultrasound helpful to } \\
\text { differentiate } \\
\text { - More likely to be viable }\end{array}$ & $\begin{array}{l}\text { - Ectopic pregnancy implanted } \\
\text { in the intramural portion of } \\
\text { tube } \\
\text { - Separated from endometrial } \\
\text { canal by thin band of } \\
\text { endometrium } \\
\text { - Higher mortality rate due to } \\
\text { possible rupture }\end{array}$ \\
\hline
\end{tabular}

RIT 2017

\section{Cervical pregnancy (cont'd)}

- Comparison of gestational sac and abortion in progress

- Gestational sac (black arrow) containing fetal pole within cervix (Image $A$ and $B$ )

- Abortion in progess. Differentiated from cervical pregnancy by sliding sign - gestational sac (white arrow) that can be manipulated by transducer (Image C)

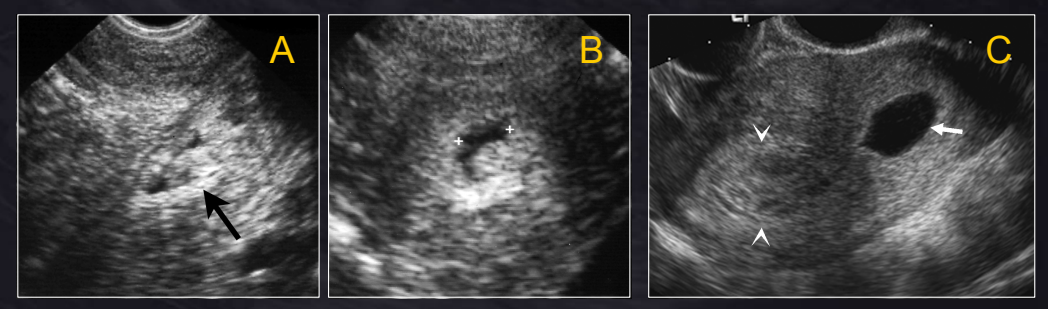

RIT 2017 
Management options for Cervical pregnancy

- Hysterectomy

- Methotrexate

- Uterine artery embolization

Dilation and curettage results in excessive hemorrhage

Gestational sac containing fetal pole within anterior wall of uterus underlying scar (arrow) on grey scale (Image A) and power Doppler (Image B)

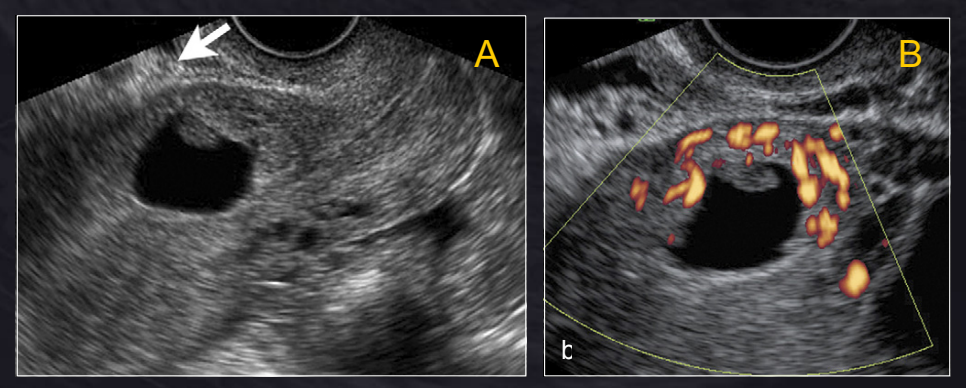

Scar pregnancy

- + h/o C Section

- Rare: $0.15 \%$

- Implantation through sinus tract within anterior uterine wall created during prior surgery

- Criteria for diagnosis

- Empty uterus and cervical canal

- Development of sac in anterior portion of lower uterine segment (large FOV on transabdominal views)

- Thinning of myometrium between bladder wall and gestational sac

RIT 2017

Scar pregnancy (cont'd)

- High risk for rupture, hemorrhage and mortality

- Treated with methotrexate or $\mathrm{KCl}$ injections 
Ovarian pregnancy

- Retention and fertilization of ova within ovary

- Uncommon, incidence of $0.5-3.0 \%$

- Criteria proposed by Spiegelberg [1978]

- Empty uterine cavity

- Serum B-HCG $\geq 1000$ IU/L

- Atypical cyst or chorionic villi within ovary

- Normal fallopian tubes

- Normalized B-HCG following therapy

- US: Adnexal cyst within or adjacent to ovary with wide echogenic rim

Heterotopic Pregnancy (cont'd)

- Typically presents with hemorrhage

- Often diagnostic challenge

- Treated by ablation of ectopic pregnancy while permitting continuation of intrauterine pregnancy
Heterotopic Pregnancy

- Intra and extrauterine pregnancy

- Incidence of 1:30,000 in 1948

- Presently up to 1:7000 secondary to IVF

- Heterotopic pregnancy occur in 1:100 cases of IVF, particularly when multiple embryos are transferred to the uterus

RIT 2017

\section{Heterotopic Pregnancy (cont'd)}

- Heterotopic pregancy (HP), with gestational sac within the right ovary (arrowhead) and uterus (arrow)
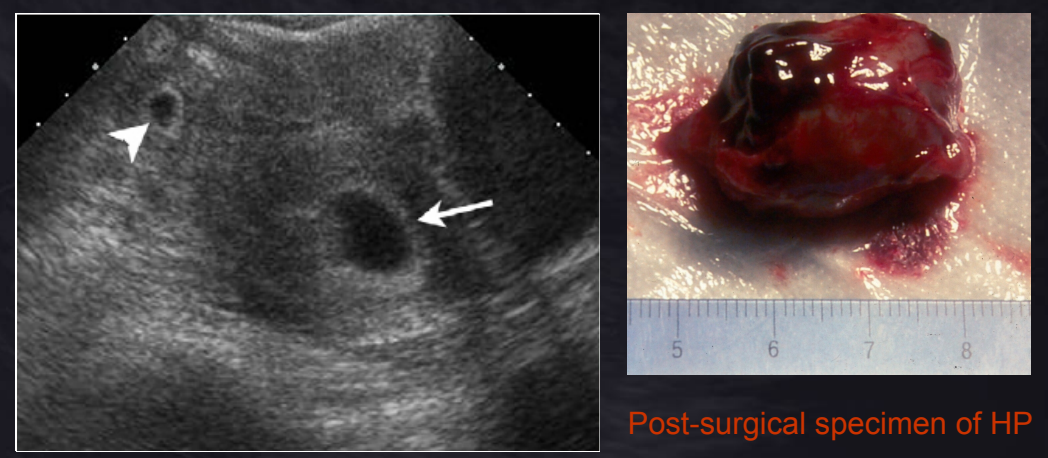
Abdominal pregnancy

most commonly results from:

a. Fertilization of ova dispersed from prior trauma or surgery

b. Rupture of tubal or ovarian pregnancy with peritoneal or intraabdominal implantation

c. In vitro fertilization

d. Post tubal ligation
Abdominal pregnancy

- Intraperitoneal implantation excluding tubal, ovarian or intraligamentous implantation

- Uncommon, incidence of 1:11,000

- Majority present with hemorrhage, often leading to mortality

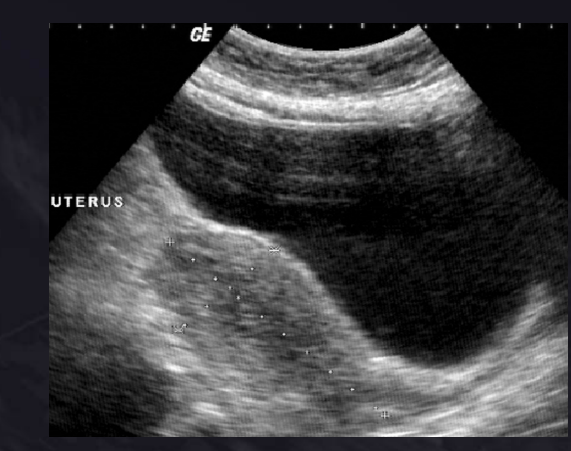

a. Laparoscopy

b. Computed

tomography

c. Abdominal Ultrasound

d. Observation
RIT 2017

\section{Abdominal pregnancy}

Transabdominal pregnancy demonstrated right lower quadrant abdominal mass containing a structure suggestive of a fetal head (Image A). Post-operative specimen (Image B) reveals non-viable fetal head.
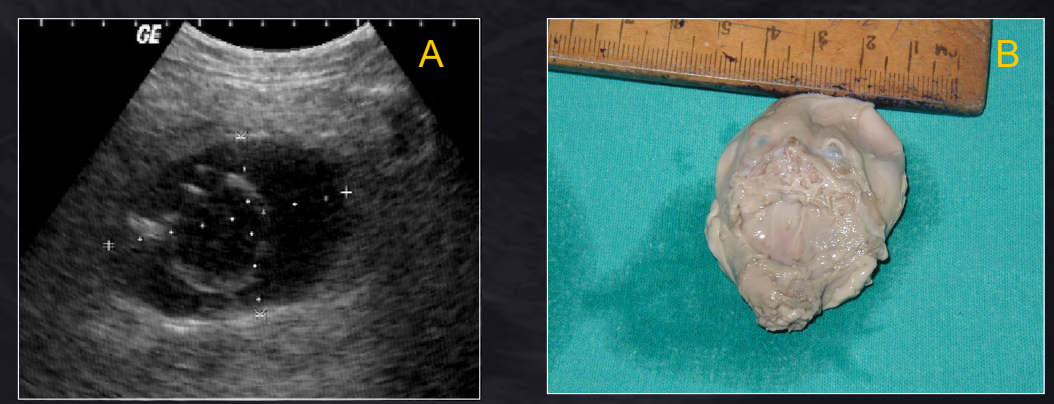
32 y.o. female, positive IUP,

6 wks 5 days clinical gestational age

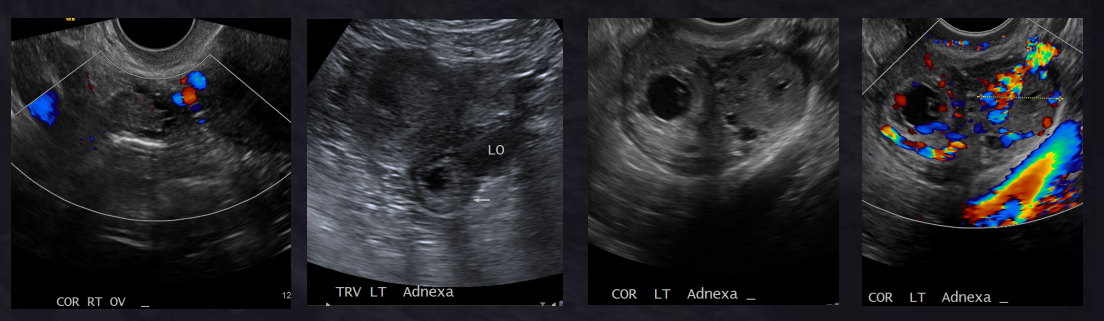

Twin Adnexal Ectopic Pregnancy

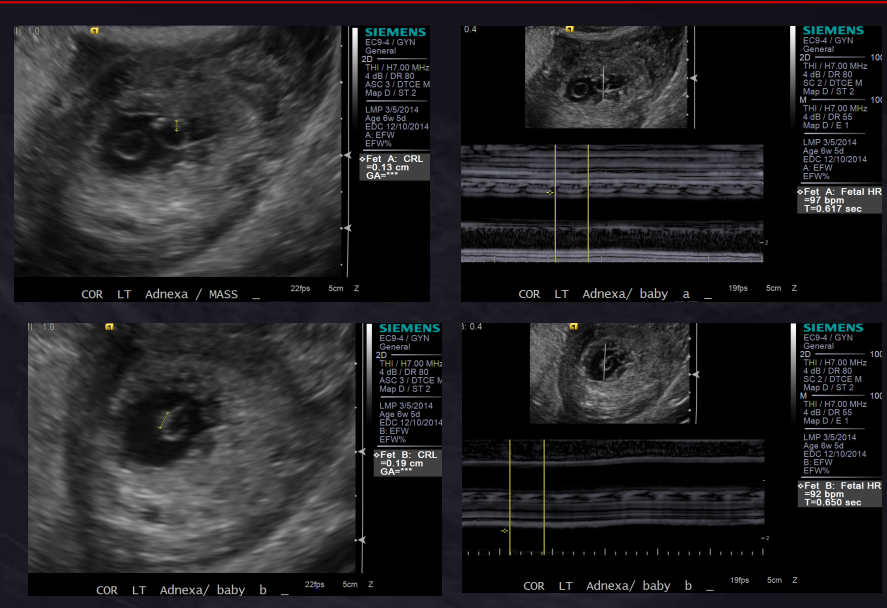

RIT 2017

\section{Unilateral Twin Ectopic Pregnancy}

Rare, 1:200 ectopic pregnancies Risk factors

Tubal surgery

Conception after tubal ligation Fertility drugs

Assisted reproductive technology
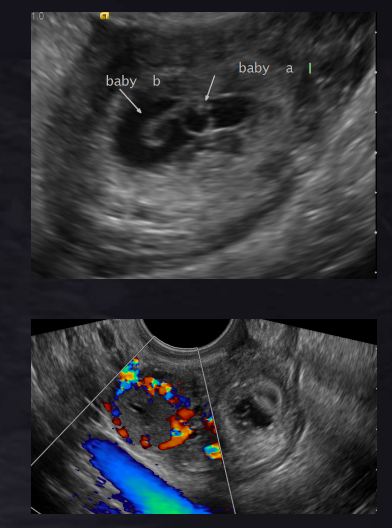

Indications for Methotrexate Therapy

- Ectopic size $<3 \mathrm{~cm}$

- Stable or rising HCG levels with peak values below 15000 IU/ml(IRP)

- No cardiac activity within gestational sac

- No active vaginal bleeding

- Ectopic pregnancy fully visualized during laproscopy 
- Poor patient compliance

- Hepatic or renal disease

- Leukocyte count of $<3000$ or platelet count of $<100,000$.

- An abnormally elevated beta-HCG without an intrauterine pregnancy should prompt search for an ectopic pregnancy

- Normal pelvic US are found in nearly onethird of ectopic pregnancies

- The most common location of an ectopic pregnancy is the fallopian tube

- Diagnostic criteria may improve specificity with US findings such as the tubal ring and interstitial line signs
Summary

- Ectopic pregnancy is the leading cause of mortality among first trimester pregnancies

- Mortality typically results from hemorrhage

- Classic triad of pelvic pain, vaginal bleeding and amenorrhea occurs in less than $50 \%$ of ectopic pregnancies

- Imaging is often needed to establish a diagnosis

RIT 2017

\section{Reference}

Bhatt S, Ghazale H, Dogra VS. Sonographic evaluation of ectopic pregnancy. Radiol Clin N Am 2007; 45: 549-560

Attar E. Endocrinology of ectopic pregnancy. Obstet Gynecol Clin N Am 2004; 31:779-94

Dogra V, Paspulati RM, BhattS. First trimester bleeding evaluation. Dogra V, Paspulati RM, Bhatts.

Timor-Tritsh IE, Monteagudo A, Matera C et al. Sonographic evaluation of cornual pregnancies treated without surgery. Obstet

- Kung FT, Lin H, Hsu TY, et al. Differential diagnosis of suspected

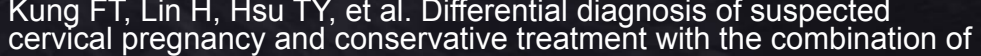
laparoscopy-assisted uterine artery ligation and hysteroscopic endocervical resection. Fertil Steril 2004; 81:1642-49.

- Spiegelberg $O$. Zur Casuistic der Ovarialschwangerschaft. Arch Gynaekol 1978; 13:73 
